\title{
Acesso aos serviços de saúde no Brasil por mulheres transexuais: uma revisão integrativa
}

\author{
Access to health services in Brazil by transsexuals women: an integrative review \\ Acceso a los servicios de salud em Brasil por parte de mujeres transexuales: uma revisión \\ integradora
}

Natália de Araújo e Silva

ORCID: https://orcid.org/0000-0001-6770-7201 Universidade Federal do Estado do Rio de Janeiro, Brasil

E-mail: natalia.silva@edu.unirio.br

Giovanna Thayla Caetano de Lima

ORCID: www.orcid.org/0000-0002-6977-7753

St. Vinzenz Krankenhaus Hanau, Alemanha

E-mail: giovannathayla@gmail.com

Eliza Cristina Macedo

ORCID: https://orcid.org/0000-0001-8824-9107

Universidade Federal do Estado do Rio de Janeiro, Brasil

E-mail: macedo.unirio@gmail.com

Cláudia Regina Ribeiro

ORCID: https://orcid.org/0000-0002-0262-0073

Universidade Federal Fluminense, Brasil

E-mail: 13cribeiro@gmail.com

Andréa Felizardo Ahmad

ORCID: https://orcid.org/0000-0002-1269-3612

Universidade Federal do Estado do Rio de Janeiro, Brasil

E-mail: andreafelizardo.enfermeira@gmail.com

Adriana Lemos

ORCID: https://orcid.org/0000-0001-9705-6200

Universidade Federal do Estado do Rio de Janeiro, Brasil

E-mail: adrianalemos@unirio.br

\begin{abstract}
Resumo
O objetivo do estudo foi analisar a produção científica brasileira sobre o acesso aos serviços de saúde por mulheres transexuais na atenção primária do SUS. Trata-se de uma revisão integrativa da literatura, realizada no período de janeiro de 2020 a janeiro de 2021, nas bases de dados Medical Literature Analysis and Retrieval System Online, Scientific Electronic Library Online, Literatura Latino-Americana e do Caribe em Ciências da Saúde, Cumulative Index to Nursing and Allied Health Literature e Banco de Dados em Enfermagem, com recorte temporal de 2013 a 2020 e selecionados a partir da questão norteadora: Como se dá o acesso aos serviços de saúde na atenção primária do SUS por mulheres transexuais? A amostra foi composta por onze artigos. Os estudos apontaram barreiras na implementação das políticas voltadas para a população trans, como, dentre outros entraves, o desrespeito ao nome social dentro das unidades de saúde. Além disso, a concentração da maior parte dos estudos na região Sudeste, prejudica uma visão geral do acesso no Brasil. A produção científica analisada evidenciou as lacunas existentes no acesso e a necessidade de instruir os profissionais de saúde da atenção primária do SUS acerca das necessidades das mulheres trans.
\end{abstract}

Palavras-chave: Transexualidade; Pessoas transgênero; Acesso aos serviços de saúde; Minorias sexuais e de gênero; Atenção primária à saúde.

\begin{abstract}
The objective is to analyse the Brazilian scientific production about the access to health services by transsexual women in primary care of the SUS. This integrative review was conducted from January 2020 to January 2021, in the database Medical Literature Analysis and Retrieval System Online, Scientific Electronic Library Online, Literatura Latino-Americana e do Caribe em Ciências da Saúde, Cumulative Index to Nursing and Allied Health Literature and Banco de Dados em Enfermagem, for the period from 2013 to 2020 and selected from the guide question: How does access to health services be given in primary care of the SUS by women transsexuals? The review was composed by eleven articles. The publications found barriers in the implementation of politics for trans population, as the social name disrespect in the health unities. Moreover, the concentration of most studies in the Southest makes harder to
\end{abstract}


make an overview of access in Brazil. The scientific production analysed showed the problems in the access and the need to educate SUS primary care health professionals about the needs of trans women.

Keywords: Transexualism; Transgender persons; Access to health services; Sexual and gender minorities; Primay health care.

\section{Resumen}

El objetivo es analizar la producción científica brasileña sobre el acceso a los servicios de salud por parte de mujeres transexuales en la atención primaria del SUS. Se trata de una revisión integradora, realizada de enero de 2020 a enero de 2021, en las bases de datos Medical Literature Analysis and Retrieval System Online, Scientific Electronic Library Online, Literatura Latino-Americana e do Caribe em Ciências da Saúde, Cumulative Index to Nursing and Allied Health Literature y Banco de Dados em Enfermagem, con un plazo de 2013 a 2020 y seleccionado de la pregunta de la guía: ¿Cómo se da el acceso a los servicios de salud en la atención primaria del SUS por las mujeres transexuales? La muestra consistió en once artículos. Los estudios señalaron barreras en la aplicación de políticas dirigidas a la población trans, como la falta de respeto por los nombres sociales dentro de las unidades de salud. Además, la concentración de la mayoría de los estudios en la región sureste perjudica una visión general del acceso en Brasil. La producción analizada eviidenció las brechas de acceso existentes y la necesidad de instruir a los profesionales de la salud de la atención primaria del SUS sobre las necessidades de las mujeres trans.

Palabras clave: Transexualidad; Personas transgénero; Accesibilidad a los servicios de salud; Minorías sexuales y de género; Atención primaria de salud.

\section{Introdução}

A transexualidade constitui um tema complexo para discussão, visto que desafia as convenções sociais fundamentadas em padrões cisheteronormativos. É entendida por Berenice Bento (2008) como a identidade de gênero das pessoas que não se identificam com as características socialmente designadas ao gênero que lhe foi determinado ao nascimento, a partir da genitália.

Patologizada, essa identidade de gênero foi incluída na categoria de transtornos mentais na Classificação Estatística Internacional de Doenças e Problemas de Saúde (CID), da Organização Mundial de Saúde (OMS), em 1990. No entanto, em sua $72^{\circ}$ Assembleia Mundial da Saúde, em 2018, a OMS retirou a transexualidade dessa categoria e a classificou em uma nova, a "condições relacionadas à saúde sexual", que constará no novo CID-11, que deverá ser adotado por todos os países até 2022. Assim, a transexualidade passa a ser compreendida pela OMS como uma incongruência de gênero, que significa ausência de conformidade, de concordância e de adequação à norma de gênero. E a nomenclatura vigente - transexualismo, cujo sufixo "ismo" caracteriza doença - será substituída por transexualidade.

Apesar desses avanços, é necessário um contínuo aporte político para que a população trans deixe de ser vislumbrada pela ótica patologizante (Silva \& Oliveira, 2013). E nessa luta, os movimentos sociais compostos por lésbicas, gays, bissexuais, travestis e transexuais (LGBT) vêm ganhando relevância ao denunciar a negação do direito à saúde imposta a essas pessoas como um desrespeito aos direitos humanos e aos princípios do próprio Sistema Único de Saúde (SUS) (Brasil, 2013), sendo a falta de preparo dos profissionais de saúde uma das causas dessa situação. Nesse contexto, é necessário compreender a dimensão da determinação social e o impacto no processo saúde-doença a que está submetida essa população desde a infância, muitas vezes, com a expulsão de casa e da escola, por exemplo. A essas exclusões agregam-se outras, consequentemente: o desemprego, a falta de acesso a bens e serviços, a alimentação adequada, a moradia e a todo tipo de violência e preconceitos.

Segundo a Política Nacional de Atenção Básica (PNAB), a atenção básica "deve ser o contato preferencial dos usuários, a principal porta de entrada e centro de comunicação da Rede de Atenção à Saúde." (Brasil, 2012), seguindo os princípios de universalidade, integralidade e equidade do SUS, bem como opondo-se à exclusão por gênero, orientação sexual, identidade de gênero, entre outros (Brasil, 2017). Porém, o acesso aos serviços é historicamente problemático para a população trans, pois as barreiras começam logo na recepção, com atendimento discriminatório, não reconhecimento do nome social, condutas inadequadas e se mantém no contato com outros profissionais que não reconhecem esta identidade como legítima por 
pautarem-se em normas rígidas que tomam a cisgeneridade e o binarismo como padrão, alijando essas pessoas dos direitos à saúde (Brasil, 2013; Gomes, Murta, Facchini \& Meneghel, 2018; Bento \& Pelúcio, 2012; Santos, Prigol, Lasmar \& Zandoná, 2020). Devido a isso, o acesso tem se dado de forma mais importante pelos serviços voltados para o processo transexualizador, ou seja, não pela atenção primária como preconiza a política, mas pela ambulatorial (Popadiuk, Oliveira \& Signorelli, 2017; Lionço, 2009). De forma especial, os profissionais de saúde que recebem um/a usuário/a que não se identifica com as normas de gênero, precisam compreender o intenso sofrimento dessas pessoas e que a exclusão social e dos serviços de saúde podem levar a diversos distúrbios de ordem psicológica, incluindo tendências suicidas (Brasil, 2013). Desta forma, é vital que os/as profissionais responsáveis pela manutenção do acesso aos serviços de saúde compreendam o contexto em que a população transexual se insere, além de como a exclusão dos serviços impacta na qualidade de vida dessas pessoas. Assim, compreender como o acesso de mulheres transexuais vem se dando nos serviços de saúde de nível primário do SUS pode contribuir para se conhecer as lacunas existentes no acesso e, dessa forma, subsidiar profissionais de saúde, bem como a introdução dessa temática em sua formação, a fim de promover modificações no manejo das demandas dessa população para que estas sejam atendidas em sua integralidade. O objetivo deste estudo foi analisar a produção científica brasileira sobre o acesso aos serviços de saúde de mulheres transexuais na atenção primária do SUS.

\section{Metodologia}

Trata-se de um estudo de natureza qualitativa, do tipo revisão integrativa da literatura, realizado no período de janeiro de 2020 a janeiro de 2021. A pesquisa qualitativa tem como foco principal a compreensão dos fenômenos a partir da correlação dos estudos, o que gera novos conhecimentos e novos significados (Pereira, Shitsuka, Parreira \& Shitsuka, 2018). A escolha desta metodologia sobre outras revisões possíveis se dá uma vez que, na revisão integrativa, é possível atingir a síntese da produção científica publicada, tal como permite identificação da aplicabilidade de seus resultados, quando significativos, na prática (Souza, Silva \& Carvalho, 2010).

O desenvolvimento da pesquisa se deu através das etapas da revisão integrativa definidas por Botelho, Cunha e Macedo (2011) sendo: identificação do tema e seleção da questão da pesquisa; estabelecimento de critérios de inclusão e exclusão; identificação dos estudos pré-selecionados e selecionados; categorização dos estudos selecionados; análise e interpretação dos resultados; apresentação da revisão/síntese do conhecimento.

Estabeleceu-se na primeira etapa do método, através do acrônimo PICo (P = População; I = fenômeno de Interesse; $\mathrm{Co}=$ Contexto), a elaboração da seguinte questão de pesquisa: Como se dá o acesso aos serviços de saúde na atenção primária do SUS (I) por mulheres (P) transexuais (Co)? 
Tabela 1. Estratégia/expressäo/string de busca.

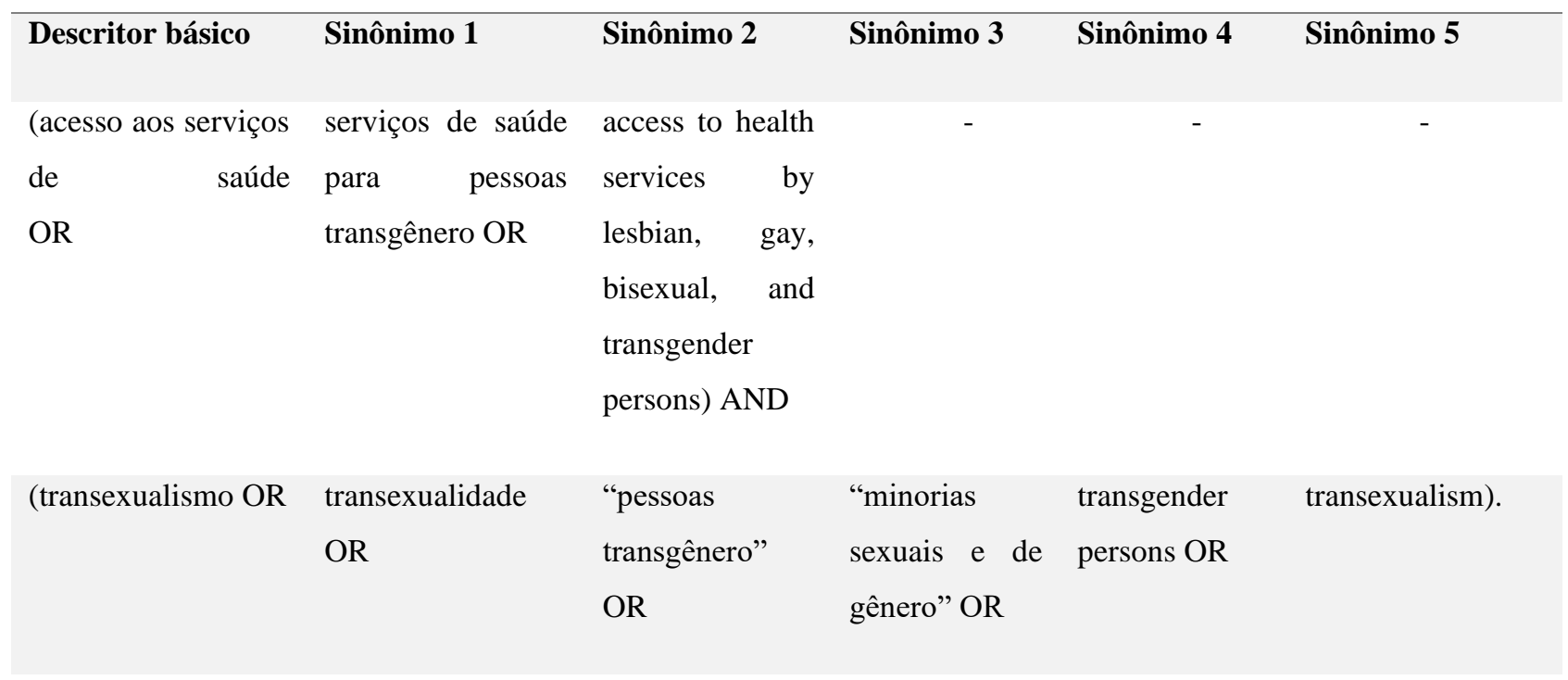

Fonte: Dados da busca.

A Tabela 1 apresenta que, para a elaboração da estratégia/equação de busca, foram utilizados os conectores lógicos/operadores booleanos AND e OR e estabelecidos os seguintes descritores/termos controlados em Ciências da Saúde (DeCS/MeSh) e descritores e palavras-chave/termos não controlados do tesauro CINAHL: acesso aos serviços de saúde; serviços de saúde para pessoas transgênero; transexualismo; transexualidade; "pessoas transgênero"; "minorias sexuais e de gênero".

Devido a constante atualização da terminologia única e rede semântica em saúde o termo controlado "transexualismo" (DeCS, 2020), em finais de 2020 passou a ser denominado "transexualidade" (DeCS/MeSH, 2020).

Na segunda etapa, foram definidos como critérios de inclusão: publicações indexadas nas bases de dados MEDLINE (Medical Literature Analysis and Retrieval System Online), SCIELO (Scientific Electronic Library Online), LILACS (Literatura Latino-Americana e do Caribe em Ciências da Saúde), CINAHL (Cumulative Index to Nursing and Allied Health Literature) e BDENF (Banco de Dados em Enfermagem); escritos nos idiomas português, inglês ou espanhol; que retratem a situação brasileira; com texto completo disponível a partir de 2013 até 2020. Foi utilizado como justificativa para o recorte temporal a data de publicação da Portaria nº 2.836, que institui, no âmbito do Sistema Único de Saúde (SUS), a Política Nacional de Saúde Integral de Lésbicas, Gays, Bissexuais, Travestis e Transexuais, em 2013. Foram excluídos os estudos repetidos e a denominada literatura cinzenta: teses e dissertações.

$\mathrm{Na}$ terceira etapa, foram identificados, por três revisores independentes, os estudos pré-selecionados através da leitura dos títulos e resumos disponibilizados nas bases de dados. Quando ocorreram dúvidas e não houve consenso entre os revisores quanto a inclusão/exclusão de registros, após a discussão, optou-se pelo desempate. Para o registro das informações relevantes oriundas do material incluído nessa revisão, as autoras elaboraram um questionário com as seguintes variáveis: base de dados, título, autoria, área, ano, local, tipo de estudo, fenômeno de interesse, Qualis CAPES, nível de evidência e qualidade do artigo.

A quarta etapa consistiu na categorização e análise dos artigos selecionados. A identificação do Qualis dos periódicos foi obtida através do Relatório Qualis Periódicos (2019). Para a classificação do nível de evidências, utilizou-se Stillwell, Fineout-Overholt, Melnyk \& Williamson, (2010), cujo os critérios podem ser analisados a seguir:

I. Evidência proveniente de uma RS ou metanálise de todos os ensaios clínicos randomizados controlados ou oriundas de diretrizes baseadas em RS de ensaios clínicos a controlados; 
II. Evidência obtida de pelo menos um ensaio clínico com aleatorização, controlado e bem delineado;

III. Evidência proveniente de um estudo bem desenhado e controlado sem aleatorização;

IV. Evidência proveniente de um estudo com desenho de caso-controle ou coorte;

V. Evidência proveniente de uma revisão sistemática de estudos qualitativos e descritivos.

VI. Evidência de um único estudo descritivo ou qualitativo;

VII.Evidência proveniente da opinião de autoridades e/ou relatórios de comissões de especialistas/peritos.

Para a análise da qualidade a ferramenta Critical Appraisal Skills Programme (Joanna Briggs Institute [JBI], 2008) que define os registros quanto ao maior ou menor risco de viés.

A quinta etapa resultou na interpretação dos dados obtidos e na sexta etapa foi realizada a síntese do conteúdo obtido.

\section{Resultados e Discussão}

Resultaram da estratégia de busca 797 artigos e após a leitura de títulos e resumos, 770 foram excluídos por não responderem à questão norteadora da revisão. Deste modo, 27 registros foram pré-selecionados para avaliação e leitura na íntegra.

Após a leitura do material completo, foram selecionados onze artigos para análise, identificados no texto pela letra A (artigo) e os números sequenciais (A1, A2, A3 [...] A11), sendo três da base de dados SCIELO, sete na LILACS, e um na BDENF. O processo pode ser acompanhado através do fluxograma PRISMA (Principais Itens para Relatar Revisões sistemáticas e Meta-análises) elaborado na Figura 1.

Figura 1. Fluxograma PRISMA.

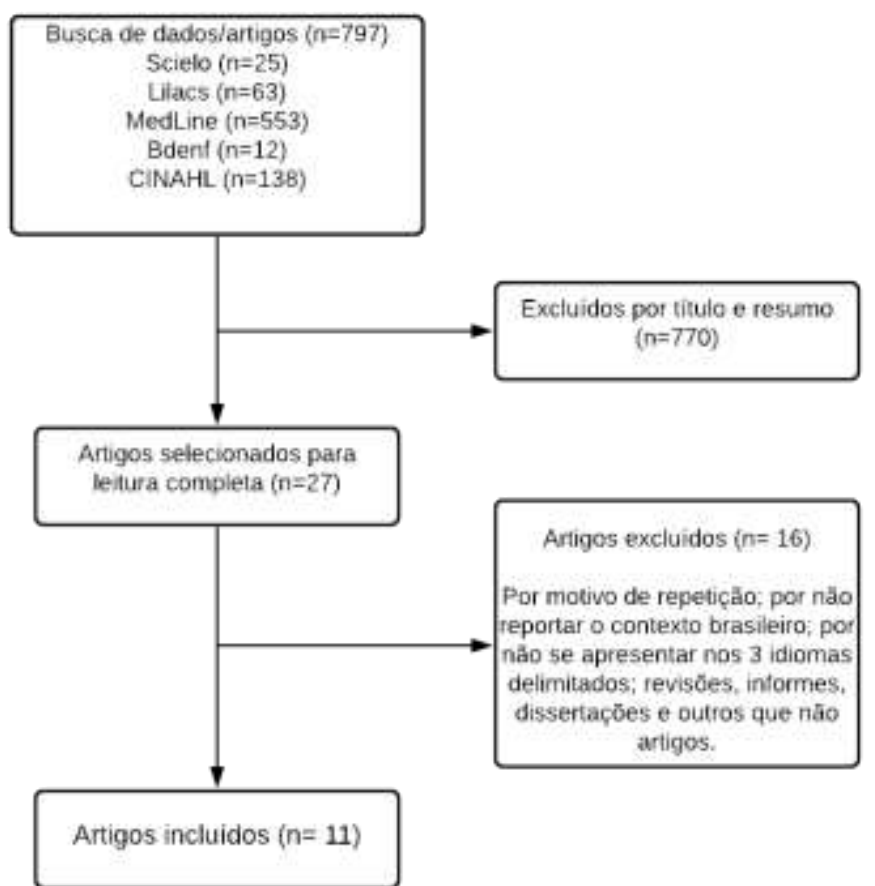

Fonte: Autores. 
No fluxograma da Figura 1, é possível observar como se deu o processo de seleção dos artigos que compõem a amostra e o número elevado de artigos excluídos por, já em seu título e resumo, apresentarem conteúdo divergente do esperado para a produção da revisão.

Quadro 1. Correlação dos estudos com base de dados título, autoria, área, ano e local.

\begin{tabular}{|c|c|c|c|c|c|c|}
\hline ID & $\begin{array}{c}\text { Base de } \\
\text { dados }\end{array}$ & Título & Autoria & Área & Ano & Local \\
\hline A1 & BDENF & $\begin{array}{c}\text { Serviços De Saúde Para } \\
\text { Lésbicas, Gays, } \\
\text { Bissexuais E } \\
\text { Travestis/Transexuais }\end{array}$ & $\begin{array}{l}\text { OLIVEIRA, Geane } \\
\text { et al }\end{array}$ & Enfermagem & 2018 & PB \\
\hline A2 & LILACS & $\begin{array}{c}\text { Dificuldades vividas por } \\
\text { pessoas trans } \\
\text { no acesso ao Sistema } \\
\text { Único de Saúde }\end{array}$ & $\begin{array}{c}\text { ROCON, Pablo et } \\
\text { al }\end{array}$ & Saúde Coletiva & 2016 & ES \\
\hline A3 & LILACS & $\begin{array}{c}\text { Acesso de lésbicas, gays, } \\
\text { bissexuais e } \\
\text { travestis/transexuais às } \\
\text { Unidades Básicas de } \\
\text { Saúde da Família }\end{array}$ & $\begin{array}{l}\text { OLIVEIRA, Geane } \\
\text { et al }\end{array}$ & Enfermagem & 2018 & PB \\
\hline A4 & LILACS & $\begin{array}{c}\text { Diversidade de gênero e } \\
\text { acesso ao sistema único } \\
\text { de saúde }\end{array}$ & $\begin{array}{c}\text { FERREIRA, } \\
\text { Breno; PEDROSA, } \\
\text { José; } \\
\text { NASCIMENTO, } \\
\text { Elaine; }\end{array}$ & Saúde coletiva & 2018 & PI \\
\hline A5 & SCIELO & $\begin{array}{l}\text { Discursos sobre o cuidado } \\
\text { em saúde de Lésbicas, } \\
\text { Gays, Bissexuais, } \\
\text { Travestis, Transexuais } \\
\text { (LGBT) entre médicas(os) } \\
\text { da Estratégia Saúde da } \\
\text { Família }\end{array}$ & $\begin{array}{l}\text { PAULINO, Danilo; } \\
\text { RASERA, } \\
\text { Emerson; } \\
\text { TEIXEIRA, Flavia; }\end{array}$ & Medicina & 2019 & MG \\
\hline A6 & SCIELO & $\begin{array}{l}\text { Experiências de acesso de } \\
\text { mulheres trans/travestis } \\
\text { aos serviços de saúde: } \\
\text { avanços, limites e tensões }\end{array}$ & $\begin{array}{l}\text { MONTEIRO, } \\
\text { Simone; } \\
\text { BRIGEIRO, } \\
\text { Mauro; }\end{array}$ & Saúde Coletiva & 2019 & RJ \\
\hline A7 & LILACS & $\begin{array}{l}\text { (Re)escrevendo roteiros } \\
\text { (in)visíveis: a trajetória de } \\
\text { mulheres transgênero nas } \\
\text { políticas públicas de } \\
\text { saúde. }\end{array}$ & $\begin{array}{l}\text { OLIVEIRA, } \\
\text { Itauane; } \\
\text { ROMANINI, } \\
\text { Moises. }\end{array}$ & Psicologia & 2020 & $\mathrm{RS}$ \\
\hline A8 & LILACS & $\begin{array}{c}\text { Avaliação da } \\
\text { implementação da Política } \\
\text { Nacional de Saúde } \\
\text { Integral à população } \\
\text { LGBT em um município } \\
\text { da região Sudeste do } \\
\text { Brasil }\end{array}$ & $\begin{array}{l}\text { GUIMARÃES, } \\
\text { Nilo Plantiko et al }\end{array}$ & Enfermagem & 2020 & ES \\
\hline A9 & LILACS & $\begin{array}{l}\text { Política nacional de saúde } \\
\text { integral LGBT: o que } \\
\text { ocorre na prática sob o } \\
\text { prisma de usuários (as) e }\end{array}$ & $\begin{array}{c}\text { NOGUEIRA, } \\
\text { Francisco Jander de } \\
\text { Sousa; ARAGÃO, } \\
\text { Thalia Ariadne }\end{array}$ & $\begin{array}{l}\text { Medicina / } \\
\text { psicologia }\end{array}$ & 2019 & PI \\
\hline
\end{tabular}




\begin{tabular}{|c|c|c|c|c|c|c|}
\hline & & profissionais de saúde & Peña. & & & \\
\hline A10 & LILACS & $\begin{array}{l}\text { Travestis e transexuais: } \\
\text { despindo as percepções } \\
\text { acerca do acesso e } \\
\text { assistência em saúde }\end{array}$ & $\begin{array}{l}\text { LOVISON, Robson } \\
\text { et al }\end{array}$ & Enfermagem & 2019 & $\mathrm{SC}$ \\
\hline A11 & SCIELO & $\begin{array}{c}\text { Desafios enfrentados por } \\
\text { pessoas trans para acessar } \\
\text { o processo } \\
\text { transexualizador do } \\
\text { Sistema Único de Saúde }\end{array}$ & $\begin{array}{l}\text { ROCON, Pablo } \\
\text { Cardozo et al }\end{array}$ & Saúde coletiva & 2019 & $\begin{array}{l}\text { Sudeste } \\
\text { (não foi } \\
\text { especifica } \\
\text { do o } \\
\text { estado) }\end{array}$ \\
\hline
\end{tabular}

Fonte: Dados da pesquisa.

É possível observar no Quadro 1, que as áreas de publicação predominantes foram a enfermagem e saúde coletiva, com ano de publicação compreendido entre 2016 e 2020. Isso reflete que, por mais que a Política Nacional de Saúde Integral de Lésbicas, Gays, Bissexuais, Travestis e Transexuais tenha sido preconizada em 2013, levou um tempo para que estudos relevantes para a atual revisão fossem produzidos.

A amostra foi composta por estudos realizados na Paraíba, Espírito Santo, Piauí, Minas Gerais, Rio Grande do Sul, Santa Catarina, Rio de Janeiro e um estado não especificado da região Sudeste. Nota-se que o Sudeste se sobressai com 45,45\%, com três dos quatro estados da região, seguido do Nordeste, com 36,36\%, porém, com menos diversidade de estados, com estudos concentrados apenas no Piauí e na Paraíba. Esses números são justificados visto que estas são as regiões mais populosas do Brasil. Nenhuma pesquisa da amostra foi realizada na região Norte e Centro-Oeste, o que dificulta uma visão geral do acesso de mulheres trans à atenção básica do SUS.

Quadro 2. Correlação dos estudos com tipo de estudo, fenômeno de interesse (I), Qualis CAPES, nível de evidência e qualidade do artigo.

\begin{tabular}{|c|c|c|c|c|c|}
\hline ID & Tipo de Estudo & Fenômeno de interesse & Qualis CAPES & Nível de evidência & Qualidade do artigo \\
\hline A1 & Quantiqualitativo & Acesso dificultado & B4 & VI & Baixo risco de viés \\
\hline A2 & Qualitativo & Acesso dificultado & A3 & VI & Baixo risco de viés \\
\hline A3 & Qualitativo & Acesso dificultado & B4 & VI & Baixo risco de viés \\
\hline A4 & Qualitativo & Acesso dificultado & $\mathrm{B} 2$ & VI & Baixo risco de viés \\
\hline A5 & Qualitativo & $\begin{array}{l}\text { Profissionais afirmam não } \\
\text { haver dificuldade no acesso. } \\
\text { Porém, foi possível notar } \\
\text { desconhecimento em suas } \\
\text { falas. }\end{array}$ & A4 & VI & Baixo risco de viés \\
\hline A6 & Qualitativo & $\begin{array}{l}\text { Segundo as entrevistadas, o } \\
\text { acesso não é dificultado pelo } \\
\text { fato de serem mulheres trans. }\end{array}$ & A3 & VI & Baixo risco de viés \\
\hline A7 & Qualitativo & Acesso dificultado & B3 & VI & Baixo risco de viés \\
\hline
\end{tabular}




\begin{tabular}{|c|c|c|c|c|c|}
\hline \multirow{2}{*}{ A8 } & Qualitativo & $\begin{array}{c}\text { Despreparo e } \\
\text { desconhecimento acerca da } \\
\text { Política Nacional de Saúde } \\
\text { Integral de Lésbicas, Gays, } \\
\text { Bissexuais, Travestis e } \\
\text { Transexuais. }\end{array}$ & B3 & VI & Baixo risco de viés \\
\hline A9 & Qualitativo & Dificuldade no acesso e & & & \\
distanciamento dos & profissionais da realidade da & A3 & VI & Baixo risco de viés \\
\hline A10 & Qualitativo & Discriminação presente no & B1 & VI & Baixo risco de viés \\
\hline A11 & Qualitativo & Dificuldade no acesso. & A4 & VI & Baixo risco de viés \\
\hline
\end{tabular}

Fonte: Dados da pesquisa.

O Quadro 2 apresenta os tipos de estudos utilizados, o fenômeno de interesse analisado, Qualis Capes, o nível de evidência e a qualidade dos artigos. Dos onze estudos que compõem a amostra, dez possuem abordagem qualitativa e um com abordagem quantiqualitativa, sendo sete realizados com mulheres trans usuárias da atenção primária do SUS, três realizados com profissionais da atenção básica e um realizado com ambos, usuárias e profissionais. Em 90,9\% dos artigos foi possível notar falhas que dificultam o acesso de mulheres trans ao sistema único de saúde. Quanto a classificação do Qualis, três artigos foram classificados como Qualis A3, dois como Qualis A4, um como Qualis B1, um como Qualis B2, dois como Qualis B3 e dois como Qualis B4. Sobre o nível de evidência e qualidade, os artigos foram classificados no nível seis, por seguirem abordagem qualitativa, e possuem baixo risco de viés. Importante observar a prevalência de artigos que apresentam em seus resultados a discriminação presente no acesso, o dificultando.

Os profissionais de saúde identificados na amostra (A1, A5, A8 e A9), apesar das diferenças apresentadas entre si, ainda reproduzem, dentro da Unidade Básica de Saúde da Família, noções sobre pessoas trans adquiridas durante a vida de acordo com o padrão cultural onde estão inseridos. No que se refere ao acesso, de acordo com Mello et. al., a população de pessoas transexuais e travestis são as que mais encaram desafios nos serviços de saúde, não só ao buscar o processo transexualizador, mas também ao buscar atendimento rotineiro. Pereira e Chazan (2019) reforçam essa afirmação ao falarem sobre a expressão "síndrome do braço quebrado trans" (trans broken arm syndrome), utilizada para explicar a invisibilização das demandas médicas de uma pessoa trans, por profissionais de saúde assumirem que todas as questões médicas apresentadas se devem ao fato da pessoa ser trans. Esses desafios podem ter origem pela discriminação com os marcadores sociais como aparência física, cor/raça, classe e nome social.

Ainda nos estudos realizados com os profissionais de saúde (A1, A5, A8 e A9), foi identificado que a necessidade de um atendimento equânime é reconhecida, porém, ainda rodeada de estigmas acerca da prática sexual dessa comunidade, como a concepção equivocada desta ser mais vulnerável a infecções e doenças sexualmente transmissíveis devido à prática de sexo desprotegido e múltiplos parceiros sexuais. Essa conduta também pode ser vista em pessoas cis e heterossexuais, entretanto, o desvio dos padrões estabelecidos socialmente resulta em associações discriminatórias, mascarando os reais obstáculos enfrentados pela população, como a falta de acesso e a prostituição como produto da exclusão social. A falta de investigação 
acerca das demandas da comunidade trans advém de uma cultura repleta de estigmas e da cisheteronormatização do atendimento de saúde (Oliveira, Nogueira, Costa, Silva \& Almeida, 2018).

Notou-se ainda a crença de que o problema no acesso de pessoas trans aos serviços de saúde se dá devido ao comportamento recluso desta. Não há, portanto, o questionamento acerca das razões que levaram essas pessoas a aderirem uma postura mais recolhida e a não falarem diretamente sobre suas questões sexuais, reprodutivas ou sobre qualquer outro problema de saúde, sendo este, muitas das vezes, resultado de uma fraca interação entre profissional e paciente.

Apesar da Carta dos Direitos dos Usuários do SUS, lançada em 2009 por meio da portaria $\mathrm{n}^{\circ} 1820$, tornar obrigatório um campo nos documentos de identificação para preenchimento com o nome social do usuário, caso o tenha, todos os estudos realizados com usuárias da atenção primária do SUS (A2, A3, A4, A6, A7, A9, A10 e A11), revelam o desrespeito a essa norma na prática. Embora este seja um facilitador do acesso, há mulheres trans que ainda são chamadas verbalmente pelo nome de registro, mesmo com o nome social evidenciado no prontuário. Isso, somado aos olhares discriminatórios e falta de sigilo profissional, resulta na falta de procura pelo atendimento e omissão de identidade de gênero e orientação sexual nas consultas.

Além disso, segundo o estudo realizado no interior do Rio Grande do Sul (A7), o diagnóstico ainda obrigatório para realizações de cirurgias e procedimentos transgenitalizadores, reforça o domínio da cisheteronormatividade, colocando o destino de pessoas trans a serem decididos por médicos, em sua maioria cisgêneros e heterossexuais. A análise é feita a partir da visão estereotipada e construída socialmente do que é ser homem e mulher. Na amostra, em sua quase totalidade, mulheres relatam sentirem-se mais seguras e acolhidas em atendimentos no serviço ambulatorial de atenção especializada no processo transexualizador, porém, a necessidade de transpassar pela atenção primária é desmotivadora, visto que nela são constantemente avaliadas e não há tanto preparo dos profissionais. O processo burocrático do diagnóstico e a discriminação encontrada dentro das unidades de saúde, são dificultadores de acesso e permanência no tratamento assistido, fazendo com que grande parte das mulheres trans busquem soluções mais rápidas e baratas, porém perigosas, como aplicação de silicone industrial sem o amparo de um profissional qualificado (Rocon, Rodrigues, Zamboni \& Pedrini, 2016).

Devido à grande demanda e após a I Conferência Estadual de Gays, Lésbicas, Bissexuais e Transexuais, realizada em 2008 - que preconizava a criação de um serviço de saúde específico para atenção à essa população - (Gianna \& Shimma, 2018), foi criado em 2009, na cidade de São Paulo, um Ambulatório de Saúde Integral a Travestis e Transexuais com profissionais de diversas áreas como dermatologistas e urologistas, atuando de maneira multidisciplinar no atendimento às demandas gerais dessa população (MELLO et al., 2011). Apesar do avanço, este está longe de ser um cenário ideal, visto que, além de não estar disponível em todo país, não garante mudanças e o acesso a essa população à atenção primária, como determina a Política Nacional de Saúde Integral LGBT. Assim, permanecem os entraves, como apontam Lovison, Ascari, Zocche, Durand e Ascari (2019) ao afirmarem que

A incoerência entre as Leis e políticas elaboradas e sua efetivação prática, é resultado, principalmente da dificuldade em se quebrar os paradigmas culturais, ou seja, se avançou um pouco na construção legal para garantia dos direitos, mas quase nada na quebra de preconceitos que se sobrepõem às leis no cotidiano da sociedade e seus grupos, incluindo a saúde.

Podemos acrescentar que o município de Niterói foi o precursor no Estado do Rio de Janeiro ao criar, em 2018, um ambulatório de atendimento especializado no cuidado em saúde da população trans (Fundação municipal de saúde de Niterói, 2020).

Contudo, ainda que a grande maioria das usuárias entrevistadas nos estudos relatem muitos problemas com relação ao atendimento, uma parcela, representada no estudo A6, ainda acredita que estes não são impedimentos ao acesso no sistema de saúde. Essas mulheres afirmam não sofrerem a discriminação por muitos mencionada e fazem referência às condições de 
violência em que viviam nas décadas passadas, considerando o atendimento na saúde atual, mesmo que problemático em algumas questões específicas, como desrespeito ao nome social, e gerais, como problemas estruturais do Sistema Único de Saúde (SUS), um avanço na luta pela equidade.

\section{Conclusão}

De acordo com as produções científicas brasileiras analisadas, enquanto profissionais de saúde afirmam não haver diferença de tratamento e responsabilizam as mulheres trans e as travestis pela dificuldade no acesso, ignorando as questões discriminatórias que essas pessoas enfrentam durante a vida, as usuárias do Sistema Único de Saúde (SUS), em sua maioria, reforçam a noção do mau atendimento e preconceito nas unidades de saúde. A combinação da falta de capacitação dos profissionais e o entendimento cultural destes, substancia a abstenção da procura pelo conhecimento das demandas específicas de cada usuária e da responsabilidade pela exclusão, restringindo o acesso, muitas vezes, à realização de exames de prevenção às infecções sexualmente transmissíveis (IST) e distribuição de preservativos.

Em contrapartida, a ideia de que o atendimento atual representa avanço, comparada ao passado, é disseminada por uma parcela de mulheres trans e travestis. Acredita-se que esses depoimentos se diferenciam dos outros devido a fatores como a idade das entrevistadas e a cidade onde residem, ratificando a ideia de que a promoção de um cuidado mais qualificado e eficiente depende do modo de pensar e agir dos profissionais, sendo este dependente do investimento em capacitação das Secretarias da Saúde de cada estado.

No entanto, por mais que seja necessário o fornecimento de cursos, cartilhas e atividades para os trabalhadores, apenas isso não se mostra eficaz, sendo indispensável a implementação de discussões sobre gênero e sexualidade e suas múltiplas formas de expressão na formação profissional em saúde e na educação permanente dos profissionais, a fim de promover maior equidade no acesso à saúde pública das minorias sexuais e de gênero.

A construção de trabalhos futuros realizados em outros estados, como na região norte e centro oeste, se faz necessária visto que, a escassez desse material dificulta a visão ampla do acesso de mulheres trans na atenção primária do SUS no Brasil. Além disso, sugere-se a realização de estudos acerca do acesso em outros países da américa latina, a fim de comparar os avanços e retrocessos na implementação de políticas destinadas à população LGBT+.

\section{Referências}

Bento, B. (2008). O que é transexualidade? Brasiliense.

Bento, B. \& Pelúcio, L. (2012). Despatologização do Gênero: a politização das identidades abjetas. Estudos Feministas, 20 (2), 559 - 568. https://doi.org/10.1590/S0104-026X2012000200017.

Botelho, L. L. R., Cunha, C. C. A. \& Macedo, M. (2011). O Método da Revisão Integrativa nos Estudos Organizacionais. Gestão e Sociedade, 5 (11), 121 136. https://doi.org/10.21171/ges.v5i11.1220

Brasil. Ministério da Saúde. Secretaria de Atenção à Saúde. Departamento de Atenção Básica. (2012). Política Nacional de Atenção Básica. Brasília: Ministério da Saúde. http://189.28.128.100/dab/docs/publicacoes/geral/pnab.pdf.

Brasil. Ministério da Saúde. Secretaria de Gestão Estratégica e Participativa. Departamento de Apoio à Gestão Participativa. (2013). Política Nacional de Saúde Integral de Lésbicas, Gays, Bissexuais, Travestis e Transexuais. Brasília: $1 . \quad$ ed., $1 . \quad$ reimp. $32 \quad$ p.: il. https://bvsms.saude.gov.br/bvs/publicacoes/politica_nacional_saude_lesbicas_gays.pdf

Brasil. Ministério da Saúde. (2017). Portaria $n^{o} 2.436$ de 21 de setembro de 2017. Brasília: Diário Oficial da República Federativa do Brasil. https://bvsms.saude.gov.br/bvs/saudelegis/gm/2017/prt2436_22_09_2017.html

Fundação municipal de saúde de niterói. (2020). Ambulatório de saúde para travestis e transexuais faz 1 ano. http://www.saude.niteroi.rj.gov.br/index.php?option=com_content\&view=article\&id=940\%3Atravs-1\&Itemid=1

Gianna, M. C., Martins, R. B. \& Shimma, E. (2018). Ambulatório de Saúde Integral para Travestis e Transexuais: desafios e realizações. Diversidade Sexual e de Gênero, 19(2), p. 98-104. https://docs.bvsalud.org/biblioref/2019/09/1016654/bis-v19n2-diversidade-98-104.pdf. 
Gomes, R., Murta, D., Facchini, R. \& Meneghel, S. N. (2018). Gênero, direitos sexuais e suas implicações na saúde. Ciência \& Saúde Coletiva, 23 (6), p. 1997-2005. https://doi.org/10.1590/1413-81232018236.04872018.

Joanna Briggs Institute. (2014). Joanna Briggs Institute Qualitative Assessment and Review Instrument (QARI). JBI QARI Data Extraction Form for Interpretive \& Critical Research.

Mello, L., Perilo, M., Braz, C. A. \& Pedrosa, C. (2011). Políticas de saúde para lésbicas, gays, bissexuais, travestis e transexuais no Brasil: em busca de universalidade, integralidade e equidade. Sexualidad, Salud y Sociedad, (9), 7-28. https://dx.doi.org/10.1590/S1984-64872011000400002

Ministério da saúde. Gabinete do Ministro. (2009). Portaria $n^{o} 1.820$, de 13 de agosto de 2009 . Brasília. https://bvsms.saude.gov.br/bvs/saudelegis/gm/2009/prt1820_13_08_2009.html

Monteiro, S. \& Brigeiro, M. (2019). Experiências de acesso de mulheres trans/ travestis aos serviços de saúde: avanços, limites e tensões. Cadernos de Saúde Pública. 21 (8), 1-12. https://doi.org/10.1590/0102-311X00111318

Lionço, T. (2009). Atenção integral à saúde e diversidade sexual no processo transexualizador do SUS: avanços, impasses, desafios. Physis: Revista de Saúde Coletiva, 19 (1), 43-63. https://doi.org/10.1590/S0103-73312009000100004.

Lovison, R., Ascari, T. M., Zocche, D. A. A., Durand, M. K. \& Ascari, R. A. (2019). Travestis e transexuais: despindo as percepções acerca do acesso e assistência em saúde. Enfermagem em Foco, 10 (5), 167-172. https://pesquisa.bvsalud.org/portal/resource/pt/biblio-1097701

Ministério da Educação. Coordenação de Aperfeiçoamento de Pessoal de Nível Superior. Diretoria de Avaliação. (2019). Relatório do Qualis Periódicos Área 20: ENFERMAGEM. https://www.gov.br/capes/pt-br/centrais-de-conteudo/relatorio-qualis-enfermagem-pdf.

Oliveira, G. S., Nogueira, G. A., Costa, G. P. O., Silva, F. V \& Almeida, S. A. (2018). Access by lesbians, gays, bisexuals and transvestites/transsexuals to the Basic Family Health Units. Revista da Rede de Enfermagem do Nordeste, (19), 1 - 7. https://doi.org/10.15253/2175-6783.2018193295

Oliveira, I. \& Romanini, M. (2020). (Re)writing (in)visible scripts: The trajectory of transgender women in public health policies. Saude e Sociedade, 29(1), 1-14. https://doi.org/10.1590/s0104-12902020170961

Pereira, A. S., Shitsuka, D. M., Parreira, F. J \& Shitsuka, R. (2018). Metodologia da pesquisa científica. UFSM. : https://repositorio.ufsm.br/bitstream/handle/1/15824/Lic_Computacao_Metodologia-Pesquisa-Cientifica.pdf?sequence=1.

Pereira, L. B. C. \& Chazan, A. C. S. (2019). O acesso das pessoas transexuais e travestis à atenção primária à saúde: uma revisão integrativa. Revista Brasileira De Medicina De Família E Comunidade, 14 (41), 1795. https://doi.org/10.5712/rbmfc14(41)1795

Popadiuk, G. S., Oliveira, D. C. \& Signorelli, M.C. (2017). A Política Nacional de Saúde Integral de Lésbicas, Gays, Bissexuais e Transgêneros (LGBT) e o acesso ao Processo Transexualizador no Sistema Único de Saúde (SUS): avanços e desafios. Ciência \& Saúde Coletiva, 22 (5), 1509-1520. http://dx.doi.org/10.1590/1413-81232017225.32782016.

Rocon, P. C., Rodrigues, A., Zamboni, J. \& Pedrini, M. D. (2016). Dificuldades vividas por pessoas trans no acesso ao Sistema Único de Saúde. Ciência e Saúde Coletiva, 21 (8), 2517-2525. http://dx.doi.org/10.1590/1413-81232015218.14362015.

Santos, E. L., Prigol, A. C, Lasmar, S. A. \& Zandoná, N. S. (2020). Dificuldades enfrentadas por lésbicas, gays, bissexuais, travestis, transexuais e transgêneros ao atendimento no Programa de Saúde da Família. Research, Society and Development, 9 (9), 1-10. http://dx.doi.org/10.33448/rsd-v9i9.7024

Silva, A. L. \& Oliveira, A. A. S. (2013). Transexualidade/travestilidade na literatura brasileira: sentidos e significados. Arquivos Brasileiros De Psicologia, 65 (2), 274-287. http://pepsic.bvsalud.org/pdf/arbp/v65n2/09.pdf

Souza, M. T., Silva, M. D. \& Carvalho, R. (2010). Revisão Integrativa: o que é e como fazer. Einstein, 8 (11), $102-106$ https://www.scielo.br/pdf/eins/v8n1/pt_1679-4508-eins-8-1-0102.pdf

Stillwell, S. B., Fineout-Overholt, E., Melnyk, B. M. \& Williamson, K. M. (2010). Searching for the Evidence: Strategies to help you conduct a successful search. American Journal of Nursing (AJN), $110 \quad$ (1), $51-53 . \quad$ http://download. lww.com/wolterskluwer_vitalstream_com/PermaLink/NCNJ/A/NCNJ_546_156_2010_08_23_SADFJO_165_SDC216.pdf 